\title{
Mueller matrix decomposition of diffuse reflectance imaging in skeletal muscle
}

\author{
Xin Li and Gang Yao* \\ Department of Biological Engineering, University of Missouri, 249 Agricultural Engineering Building, Columbia, \\ Missouri 65211, USA \\ *Corresponding author: YaoG@missouri.edu
}

Received 5 January 2009; revised 10 April 2009; accepted 11 April 2009; posted 13 April 2009 (Doc. ID 105977); published 1 May 2009

\begin{abstract}
Propagation of polarized light in skeletal muscle is significantly affected by anisotropic muscle structures. To completely characterize muscle polarization properties, we acquired the whole Mueller matrix images of the diffuse reflectance. A polar decomposition algorithm was applied to extract the individual diattenuation, retardance, and depolarization images from the measured Mueller matrix. The decomposed polarization properties in muscle show distinctly different patterns from those obtained in isotropic scattering media. Stretching the prerigor muscle sample induced clear changes in the raw polarization reflectance images. However, muscle stretching induced minimal changes in the decomposed Mueller matrix images. (c) 2009 Optical Society of America

OCIS codes: $\quad 260.5430,170.3660,110.7050,120.5410$.
\end{abstract}

\section{Introduction}

Noninvasive optical techniques have been widely used to study biological tissues [1]. In addition to optical absorption and scattering, optical polarization provides a unique contrast mechanism because it is sensitive to particle morphology and other polarization properties [2-4]. Mueller matrix measurements are often use $\bar{d}$ to completely characterize sample polarization properties [5-7]. Since the 16element $(4 \times 4)$ Mueller matrix is difficult to interpret directly, decomposition methods are necessary to extract useful information from the full Mueller matrix measurements. Lu and Chipman [8] introduced the polar decomposition algorithm to derive the diattenuation, retardance, and depolarization matrices from a Mueller matrix. Swami et al. [9] explored a simplified decomposition algorithm for use in a subset $(3 \times 3)$ of the Mueller matrix measured with only linearly polarized light. Manhas et al. [10] and Ghosh et al. [11] recently extended Lu and Chipman's original method by further decomposing the total retardance into linear retardance and optical rotation.

0003-6935/09/142625-07\$15.00/0

(C) 2009 Optical Society of America
Several experimental studies have demonstrated the usefulness of such decomposition techniques in tissue characterization. Smith [12] applied polar decomposition to analyze polarization images of human skin with cancerous moles and Lupus lesions. Their results suggested that a malignant mole showed significantly less depolarization than normal tissues and Lupus showed significant retardance. Liu et al. [13] found that the decomposed polarization images revealed more tissue structural information in rat skin and melanoma tissues. Chung et al. [14] found that the decomposed depolarization and retardance images can be potentially useful for identifying precancerous lesions in oral tissue. Studies on cervix tissues [15] also showed significant difference in the diattenuation and depolarization parameters between normal and dysplasia tissues.

Skeletal muscle is an anisotropic tissue with wellorganized muscle fiber bundles. Each muscle fiber consists of many myofibrils, which appear striated due to the periodic sarcomere structure [16]. Sarcomeres are the fundamental functional unit in each muscle fiber. Our recent studies have shown that sarcomeres play important roles in modulating both unpolarized [17] and polarized [18] light propagation in 
muscle. However, the interpretation of the measured Mueller matrix in muscle is not fully understood. In this paper, we further extended our studies by applying polar decomposition to extract polarization information from the Mueller matrix images obtained in prerigor fresh skeletal muscles. In addition, because sarcomere length is closely related to force generation in skeletal muscles [16], we studied the effect of sarcomere lengths by comparing polarization images in muscle samples at their original states and when stretched along the muscle fibers. Polystyrene solutions were also studied to show the difference in isotropic media.

\section{Materials and Methods}

\section{A. Experimental Method}

Bovine sternomandibularis muscles were excised from animals immediately after slaughtering. After removing surface fat tissues, the sample was mounted on a sample holder with both ends fixed to ensure a constant sample length. A cover glass was applied on the sample surface to ensure a flat imaging surface. The muscle sample at its natural state had a width of $\sim 6 \mathrm{~cm}$ and a thickness of $\sim 12 \mathrm{~cm}$. The original length of the muscle was $11 \mathrm{~cm}$ between the two mounted ends. After a sample was imaged in its original length, the muscle was stretched and imaged again. Stretching increases the sarcomere length in prerigor muscles [16]. In this study, a muscle sample was stretched $20 \%$ over its original length along the muscle fibers. Our previous study [16] has shown that stretching over $40 \%-50 \%$ may disrupt the sarcomere structures. All measurements were finished within $1 \mathrm{~h}$ to avoid rigor-induced muscle optical property changes [16].

Figure 1 shows a schematic diagram of our experimental setup. A linearly polarized He-Ne laser was used as the light source. A polarizer (P1) was used to purify the polarization state of the incident light. The required linear or circular polarization state was generated by adjusting a variable waveplate (VW). The incident light was incident upon the sample through a $1.0 \mathrm{~mm}$ hole $\left(45^{\circ}\right)$ at the center of a $45^{\circ} \mathrm{mir}-$ ror $(M)$. The backscattered light was redirected by the mirror $(\mathrm{M})$ and imaged by a CCD camera after passing through a quarter-wave plate $(\mathrm{Q})$ and a lin-

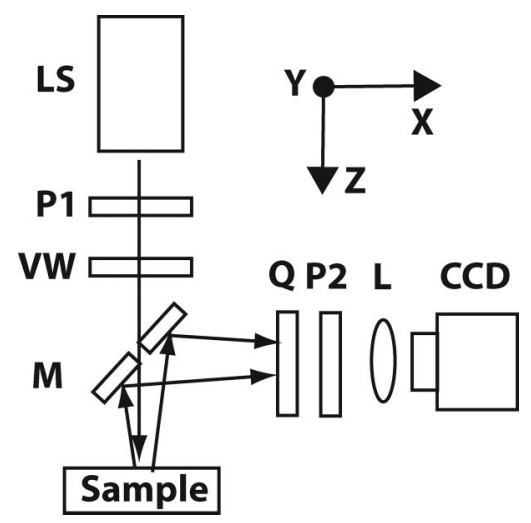

Fig. 1. Schematic of experimental setup: LS, $10 \mathrm{~mW}$ He-Ne laser; $\mathrm{P} 1, \mathrm{P} 2$, polarizers; $\mathrm{VW}$, variable wave plate; $\mathrm{M}$, mirror with a $1 \mathrm{~mm}$ hole in the center; Q, quarter-wave plate; L, imaging lens; CCD, imaging camera. The muscle sample was mounted so that the fibers were along the $y$ axis.

ear polarizer (P2). The CCD camera was an 8 bit video camera equipped with a $50 \mathrm{~mm}, f / 2.8$ imaging lens (L). The aperture of the camera lens was set to $f / 8$, corresponding to an acceptance angle of $1.2^{\circ}$. The image size was $19.9 \mathrm{~mm} \times 15.9 \mathrm{~mm}$. The entire system was carefully aligned to ensure polarization extinction ratios of $<-40$ and $<-31.7 \mathrm{~dB}$ for linearly and circularly polarized light, respectively. A reference coordinate system was defined so that the $y$ axis was aligned with the muscle fiber orientation.

The full Mueller matrices were calculated from a total of 16 polarization reflectance images recorded in the sample using the same method described before [18]. In our measurements, three different linearly polarized states were used: $H, V$, and $P$, whose polarization directions were aligned with the $x$ axis, the $y$ axis, and at $45^{\circ}$ to the $x$ axis, respectively. In addition, the right-handed circularly polarized state $R$ was also used. Thus a total of 16 images were acquired with the combination of the aforementioned four different input states (realized by the VW) and four different output states (realized by the combination of $\mathrm{P} 2$ and $\mathrm{Q}$ ). Each image was averaged over 40 times to reduce noise. The Muller matrix was calculated from these raw images:

$$
\begin{aligned}
\mathbf{M} & =\left[\begin{array}{llll}
m_{11} & m_{12} & m_{13} & m_{14} \\
m_{21} & m_{22} & m_{23} & m_{24} \\
m_{31} & m_{32} & m_{33} & m_{34} \\
m_{41} & m_{42} & m_{43} & m_{44}
\end{array}\right] \\
& =\frac{1}{2}\left[\begin{array}{cc}
H H+H V+V H+V V & H H+H V-V H-V V \\
H H-H V+V H-V V & H H-H V-V H+V V \\
2 H P+2 V P-m_{11} & 2 H P-2 V P-m_{12} \\
2 H R+2 V R-m_{11} & 2 H R-2 V R-m_{12}
\end{array}\right.
\end{aligned}
$$

$$
\begin{gathered}
2 P H+2 P V-m_{11} \\
2 P H-2 P V-m_{21} \\
4 P P-2 P H-2 P V-m_{31} \\
4 P R-2 P H-2 P V-m_{41}
\end{gathered}
$$


where each measure is represented by using two letters. The first letter denotes the input polarization state and the second letter denotes the measurement polarization state. For example, the " $H V$ " element represents a reflectance image acquired with incident polarization $H$ and detection polarization $V$. All elements are normalized against the first element $m_{11}$.

\section{B. Polar Decomposition}

The polar decomposition algorithm is used to extract several polarization parameters from the whole Mueller matrix. Theoretically, there are multiple possible decomposition families [19]. In this study, we applied the original method introduced by $\mathrm{Lu}$ and Chipman [8] and used by others [9-11] for tissue characterizations. The specific procedures applied in this study are described below.

The $4 \times 4$ Mueller matrix is decomposed into the product of three matrices corresponding to a depolar$\operatorname{izer}\left(\mathbf{M}_{\Delta}\right)$, a retarder $\left(\mathbf{M}_{R}\right)$, and a diattenuator $\left(\mathbf{M}_{D}\right)$ :

$$
\mathbf{M}=\mathbf{M}_{\Delta} \mathbf{M}_{R} \mathbf{M}_{D}
$$

With the depolarization matrix in front of the diattenuation matrix, such decomposition can produce physically possible Mueller matrices [20]. The corresponding three individual Mueller matrices have the following standard forms:

$$
\begin{aligned}
\mathbf{M}_{\Delta} & =\left[\begin{array}{cc}
1 & \overrightarrow{\mathbf{0}}^{T} \\
\overrightarrow{\mathbf{P}_{\Delta}} & \mathbf{m}_{\Delta}
\end{array}\right], \quad \mathbf{M}_{R}=\left[\begin{array}{cc}
1 & \overrightarrow{\mathbf{0}}^{T} \\
\overrightarrow{\mathbf{0}} & \mathbf{m}_{R}
\end{array}\right], \\
\mathbf{M}_{D} & =\left[\begin{array}{cc}
1 & \overrightarrow{\mathbf{D}}^{T} \\
\overrightarrow{\mathbf{D}} & \mathbf{m}_{D}
\end{array}\right],
\end{aligned}
$$

where the small case arrays $\mathbf{m}$ represent $3 \times 3$ submatrices. Specifically, the diattenuation submatrix $\mathbf{m}_{D}$ can be written as

$$
\mathbf{m}_{D}=\sqrt{1-D^{2}} \mathbf{I}+\frac{1-\sqrt{1-D^{2}}}{D^{2}} \overrightarrow{\mathbf{D}} \overrightarrow{\mathbf{D}}^{T}
$$

where $\mathbf{I}$ is the $3 \times 3$ unity matrix, $\overrightarrow{\mathbf{D}}$ is the diattenuation vector, and the diattenuation value $D$ is the length of the diattenuation vector $D=|\overrightarrow{\mathbf{D}}|$.

By multiplying the three standard matrices in Eq. (3), it is clear that the diattenuation vector can be directly derived from the measured Mueller matrix (normalized by $m_{11}$ element):

$$
\overrightarrow{\mathbf{D}}=\left[\begin{array}{lll}
m_{12} & m_{13} & m_{14}
\end{array}\right]^{T},
$$

where $m_{i j}$ is the $i$ th row and $j$ th column element of the Mueller matrix M. The diattenuation can be calculated as

$$
D=\sqrt{m_{12}^{2}+m_{13}^{2}+m_{14}^{2}}
$$

The depolarization submatrix $\mathbf{m}_{\Delta}$ is a symmetric matrix whose eigenvectors describe its depolariza- tion capabilities along the three orthogonal axes. The vector $\overrightarrow{\mathbf{P}}_{\Delta}$ describes the polarizance of the depolarizer. After obtaining $\mathbf{M}_{D}$ according to Eq. (4), the product of the depolarizing matrix $\left(\mathbf{M}_{\Delta}\right)$ and the retardance matrix $\left(\mathbf{M}_{R}\right)$ becomes

$$
\mathbf{M}_{\Delta} \mathbf{M}_{R}=\left[\begin{array}{cc}
1 & \overrightarrow{\mathbf{0}}^{T} \\
\overrightarrow{\mathbf{P}}_{\Delta} & \mathbf{m}^{\prime}
\end{array}\right]=\mathbf{M M}_{D}^{-1}
$$

where $\mathbf{m}^{\prime}=\mathbf{m}_{\Delta} \mathbf{m}_{R}$ and $\mathbf{m}$ is the $3 \times 3$ submatrix of $\mathbf{M}$. As $\mathbf{m}_{\Delta}^{T}=\mathbf{m}_{\Delta}$ and $\mathbf{m}_{\Delta}^{2}=\mathbf{m}^{\prime}\left(\mathbf{m}^{\prime}\right)^{T}, \mathbf{m}_{\Delta}$ can be derived based on the Cayley-Hamilton theorem:

$$
\begin{aligned}
\mathbf{m}_{\Delta}= & \pm\left[\left(\mathbf{m}^{\prime}\right)^{T} \mathbf{m}^{\prime}+\left(\sqrt{\lambda_{1} \lambda_{2}}+\sqrt{\lambda_{2} \lambda_{3}}+\sqrt{\lambda_{3} \lambda_{1}}\right) \mathbf{I}\right] \\
& \times\left[\left(\sqrt{\lambda_{1}}+\sqrt{\lambda_{2}}+\sqrt{\lambda_{3}}\right)\left(\mathbf{m}^{\prime}\right)^{T} \mathbf{m}^{\prime}+\sqrt{\lambda_{1} \lambda_{2} \lambda_{3}} \mathbf{I}\right],
\end{aligned}
$$

where $\lambda_{1}, \lambda_{2}$, and $\lambda_{3}$ are the eigenvalues of $\mathbf{m}^{\prime}\left(\mathbf{m}^{\prime}\right)^{T}$. The sign in Eq. (7) is the same as that of the determinant of $\mathbf{m}^{\prime}$. The total depolarization power $\Delta$ can be calculated as

$$
\Delta=1-\frac{\left|\operatorname{tr}\left(\mathbf{m}_{\Delta}\right)\right|}{3} .
$$

Once the depolarization matrix is obtained, the submatrix $\mathbf{m}_{R}$ of the retardance matrix can then be derived as

$$
\mathbf{m}_{R}=\mathbf{m}_{\Delta}^{-1} \mathbf{m}^{\prime} .
$$

The Mueller matrix $\mathbf{M}_{R}$ for a retarder can be constructed from $\mathbf{m}_{R}$ as shown in Eqs. (3). And the total retardance $R$ is calculated as

$$
R=\cos ^{-1}\left[\frac{\operatorname{tr}\left(\mathbf{M}_{R}\right)}{2}-1\right] .
$$

According to the method described in $[10,11]$, the linear retardance can be further derived by representing the retardance matrix $\mathbf{M}_{R}$ as a combination of optical rotation $\left(R_{C}\right)$ and linear retardance $\left(R_{L}\right)$. The linear retardance $R_{L}$ can be obtained from the retardance submatrix $\mathbf{m}_{R}$ as [11]

$$
\begin{aligned}
R_{L}= & \cos ^{-1}\left(\sqrt{\left[\mathbf{m}_{R(2,1)}-\mathbf{m}_{R(1,2)}\right]^{2}+\left[\mathbf{m}_{R(1,1)}+\mathbf{m}_{R(2,2)}\right]^{2}}\right. \\
& -1)
\end{aligned}
$$

where $\mathbf{m}_{R(i, j)}$ represents the $i$ th row and $j$ th column element of the retardance submatrix $\mathbf{m}_{R}$. The optical rotation is derived as

$$
2 R_{C}=\tan ^{-1}\left(\frac{\mathbf{m}_{R(2,1)}-\mathbf{m}_{R(1,2)}}{\mathbf{m}_{R(1,1)}+\mathbf{m}_{R(2,2)}}\right) .
$$

\section{Results and Discussions}

Figure 2(a) shows the raw polarization images of a muscle sample in its original length. The images 
(a) Original muscle
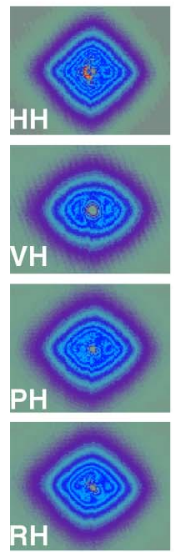

$\mathrm{RH}$
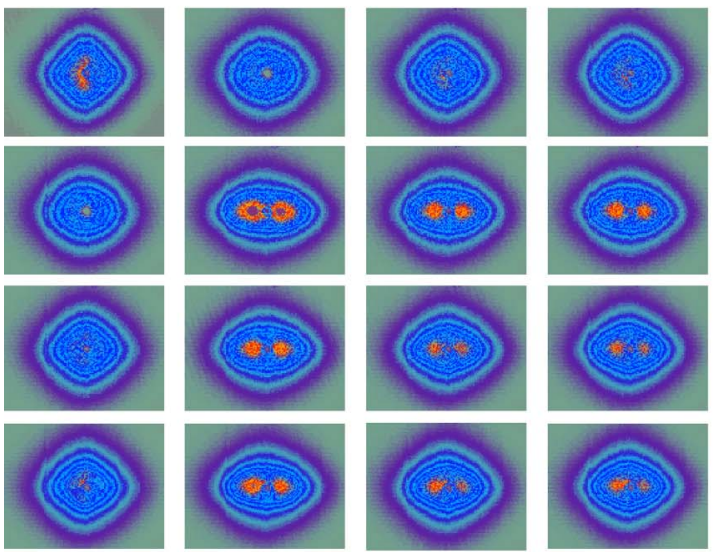

(c)Polystyrene solution
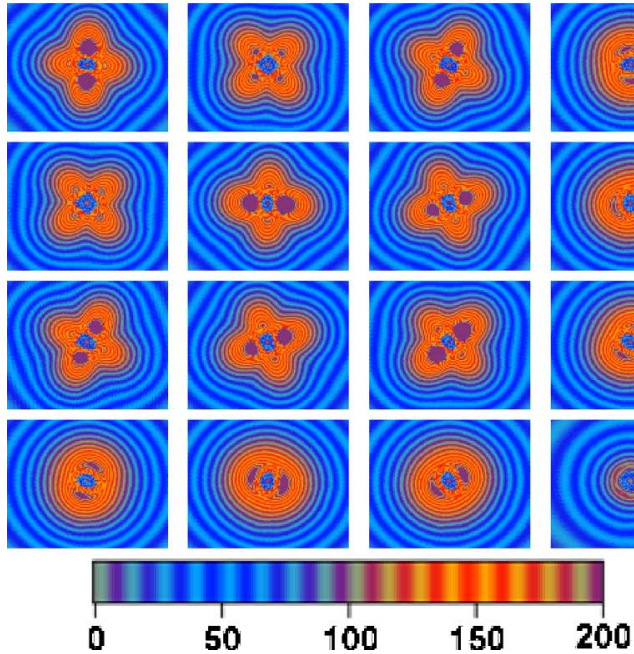
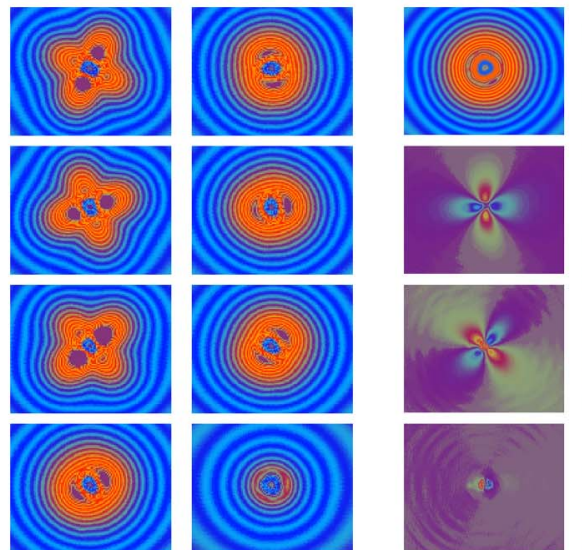

200
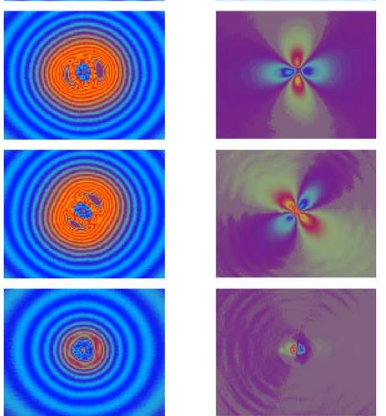
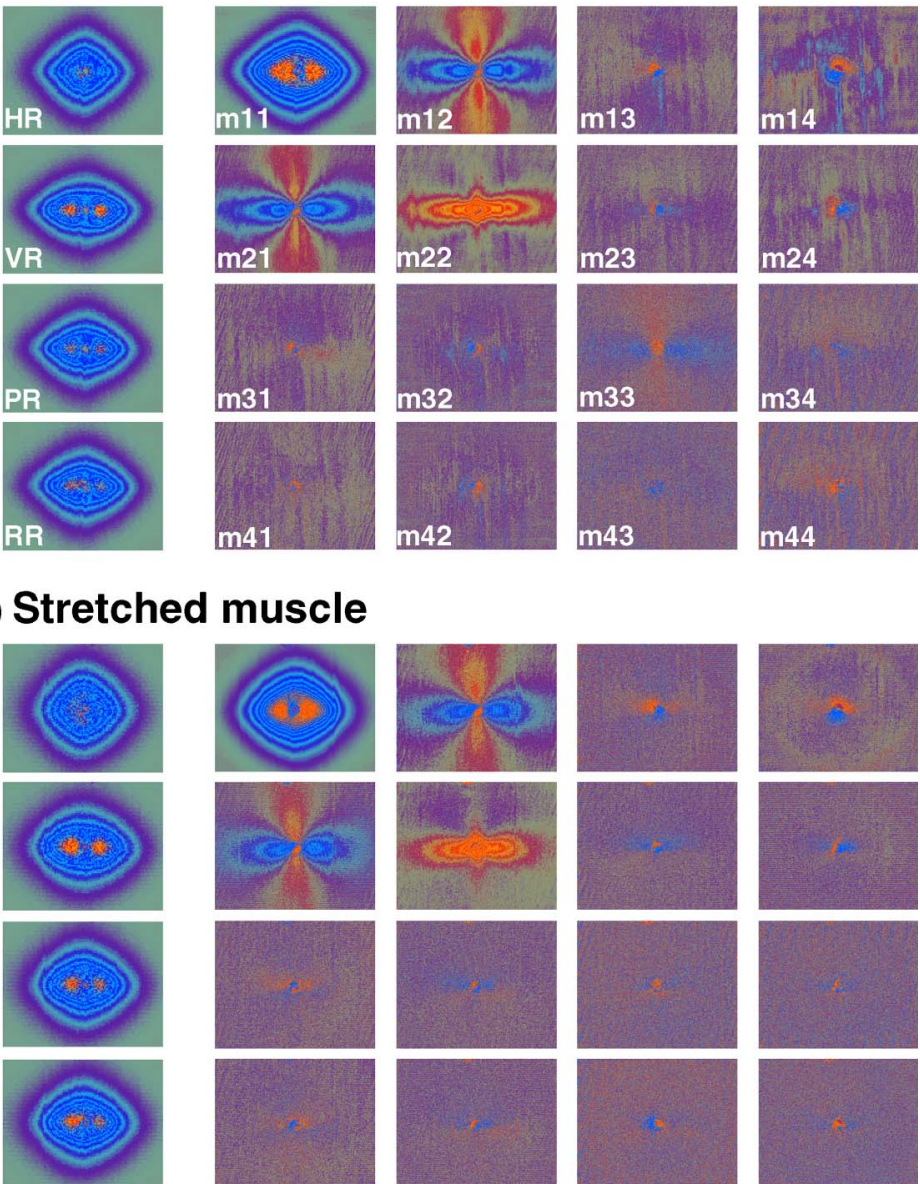

$\mathbf{m 4 4}$
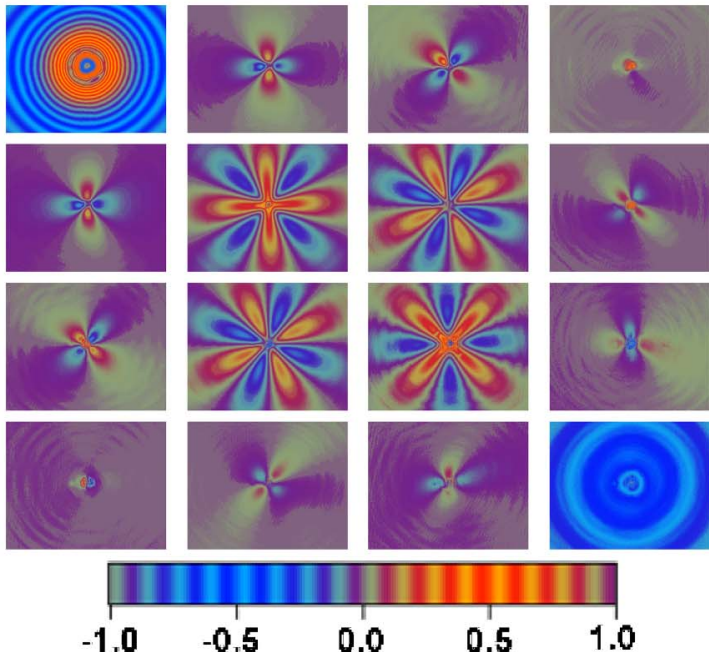

Fig. 2. (Color online) Polarization reflectance images acquired in a muscle sample at its original length and when stretched $20 \%$ along the fiber orientation (vertical direction). As a comparison, images acquired in a solution of $1.093 \mu \mathrm{m}$ polystyrenes (12\%) are also shown. The image labels are the same as shown in the first sample.

are displayed using a banded pseudocolor map so that the equi-intensity profiles can be viewed easily. The muscle fiber was along the vertical direction $y$ axis) in the image. The images are labeled using two letters: the first letter indicates the incident polarization and the second letter indicates the detection polarization. The 16 images reveal the unique rhombus-shaped reflectance patterns in skeletal 
muscles $[17,18]$. The VV image has the largest intensity and the $\mathrm{HH}$ image shows the most significant rhombuslike shape. The images in Fig. 2 show symmetric patterns along the diagonal direction. For example, the $\mathrm{HV}$ image is similar to the $\mathrm{VH}$ image and the PH image is similar to the HP image.

When the muscle sample was stretched $20 \%$ along the muscle fiber direction, the polarization reflectance images overall had similar patterns. However, most polarization images appear elongated along the stretching direction (the vertical direction). As the equi-intensity profiles are symmetric around the incident location, we applied a numerical fitting method to quantitatively describe the equi-intensity shapes using the equation

$$
\left(\frac{|x|}{a}\right)^{q}+\left(\frac{|y|}{b}\right)^{q}=1
$$

The fitting method has been described in detail elsewhere [17]. Two fitting parameters can be derived: the axes ratio $(a / b)$ and the shape parameter $q$, where $a$ and $b$ are the axis lengths of the equiintensity profile extracted at a certain distance from the incident point. The shape parameter $q=1$ indicates a rhombus and $q=2$ indicates an ellipse. As shown in Fig. 3 , stretching along the $y$ axis reduces the axes ratio in both the $\mathrm{HH}$ and the $\mathrm{VV}$ images. The VV image has a much larger change than the $\mathrm{HH}$ image. On the other hand, the $q$ parameter is larger in the stretched $\mathrm{HH}$ image, indicating the equiintensity shape has smoother corners. Similar trends exist in other images.

As a comparison, polarization images of a polystyrene solution $(1.09 \mu \mathrm{m}, 0.12 \%$ concentration) are also shown [Fig. 2(c)]. The scattering coefficient $\mu_{s}$ and anisotropy of this solution were $41 \mathrm{~cm}^{-1}$ and $0.93 \mathrm{re}$ spectively. The images acquired in the polystyrene solution show different orientations depending on the incident and detection polarizations, while the images acquired in muscles are elongated primarily along the horizontal direction. However, the center parts of the VV image are all elongated in the same way in both samples.

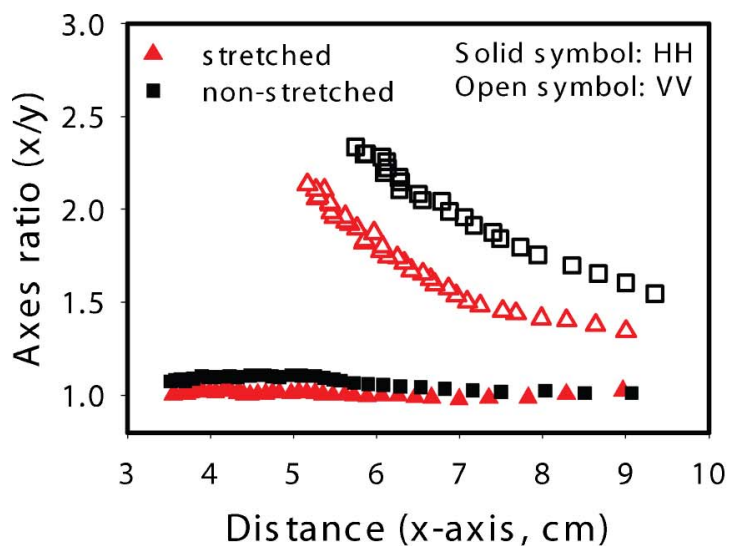

Fig. 3. (Color online) Fitted axes ratio $B$ and $q$ parameter in the HH (solid symbols) and VV (open symbols) images.

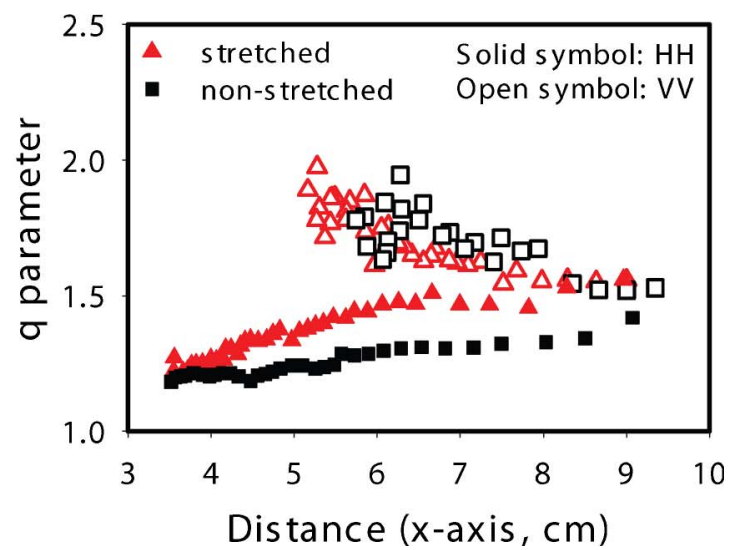

The calculated Mueller matrix images are very different in the muscle sample and the polystyrene solution. Only four elements, $m_{11}, m_{12}, m_{21}$, and $m_{22}$, show strong signals in the muscle. However, the polystyrene solution shows prominent patterns in other elements, specifically $m_{22}, m_{23}, m_{32}$, and $m_{33}$. The $m_{12}$ and $m_{21}$ images, however, appear similar in both samples. The $m_{11}$ components represent the unpolarized reflectance images. Polystyrene solution is an isotropic medium, i.e., the light scattering process is independent of the incident direction. Therefore, the corresponding equi-intensity profiles in $m_{11}$ are circular as expected. However, the skeletal muscle samples are strongly anisotropic because of the directional muscle fibers and the periodic sarcomere structures. The $m_{11}$ in muscle has a rhombuslike equi-intensity profile, as observed before [17]. Stretching the muscle induces different $m_{11}$ profiles. similar to those shown in Fig. 4 , but it does not show a clear effect on other Mueller matrix components.

Figure 4 shows the diattenuation images extracted from the $\bar{M}$ uller matrix images using Eq. (5). Diattenuation indicates the signal intensity difference between two orthogonal polarization states and its value can be from zero to 1 . The extracted diattenuation images are quite different in muscle and polystyrene solution. In the isotropic polystyrene solution, the diattenuation image has no clear pattern and the diattenuation values are close to zero at locations far away from incidence. However, the diattenuation images in muscle show a quatrefoil distribution with strong diattenuation along and perpendicular to the muscle fibers. The pattern along the muscle fiber direction has a smaller area than that perpendicular to the fibers.

Quantitatively, Fig. 4(b) shows the diattenuation values extracted $5 \mathrm{~mm}$ away from the incident point. The $0^{\circ}$ polar angle is aligned with the $x$ axis in the image. In polystyrene solution, the calculated diattenuation is less than 0.1 and does not change much with the polar angles. The reflectance in muscle shows diattenuation values between 0.1 and 0.3 . At locations perpendicular to $\left(0^{\circ}\right.$ and $\left.180^{\circ}\right)$ or parallel $\left(90^{\circ}\right.$ and $\left.270^{\circ}\right)$ with the muscle fibers, the diattenuation is higher than at other locations. In addition, the

10 May 2009 / Vol. 48, No. 14 / APPLIED OPTICS 


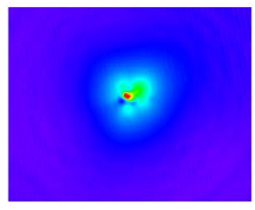

Polystyrene

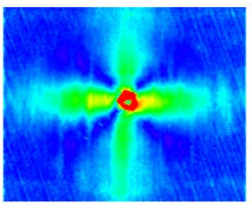

Non-stretched muscle

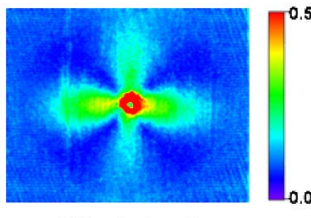

Stretched muscle

(a)

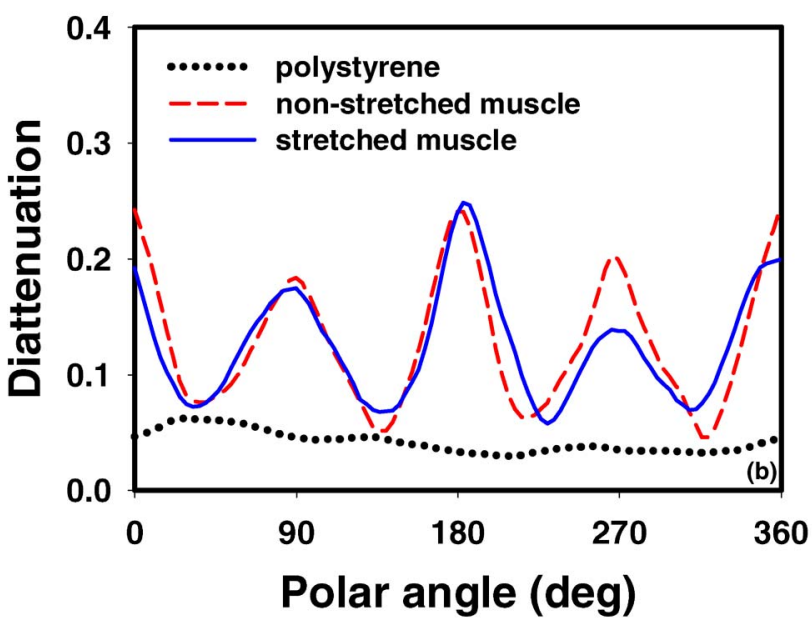

Fig. 4. (Color online) Diattenuation images obtained in muscle and polystyrene solution. The diattenuation values shown in (b) were extracted at locations $5 \mathrm{~mm}$ away from the incidence.

values are higher at $0^{\circ} / 180^{\circ}$ than at $90^{\circ} / 270^{\circ}$. Similar diattenuation patterns were observed in nonstretched and stretched muscles.

The diattenuation images in muscle are mostly determined by the $m_{12}$ element because little information exists in the $m_{13}$ and $m_{14}$ components of the muscle Mueller matrix images. Therefore, the muscle diattenuation comes mostly from linear diattenuation, specifically "horizontal" diattenuation [8]. As can be seen in the raw reflectance images (Fig. $\overline{2}$ ), more $V$-polarized incident light is backscattered along the $x$ axis; while more $H$-polarized incident light is backscattered along the $y$ axis. This phenomenon can be qualitatively explained if considering the polarization-dependent sarcomere diffraction as discussed in our recent study [18]. The sarcomeres diffract the majority of $V$-polarized light to the directions perpendicular to muscle fibers.

The depolarization power $\Delta$ describes the pure depolarizing capability of the medium. A depolarization value of 1 indicates that the sample can completely depolarize the incident light. The extracted depolarization image in polystyrene solution (Fig. 5) shows a pattern with rotational symmetry. As the distance increases from the incident point, the depolarization value increases gradually from $\sim 0.7$ to close to 1 . The incident polarized light can be depolarized by multiple scattering. As expected, the polarization state is maintained better at locations close to the incident location because the probability of multiple scattering is smaller. For those photons exiting at a large distance from the incident
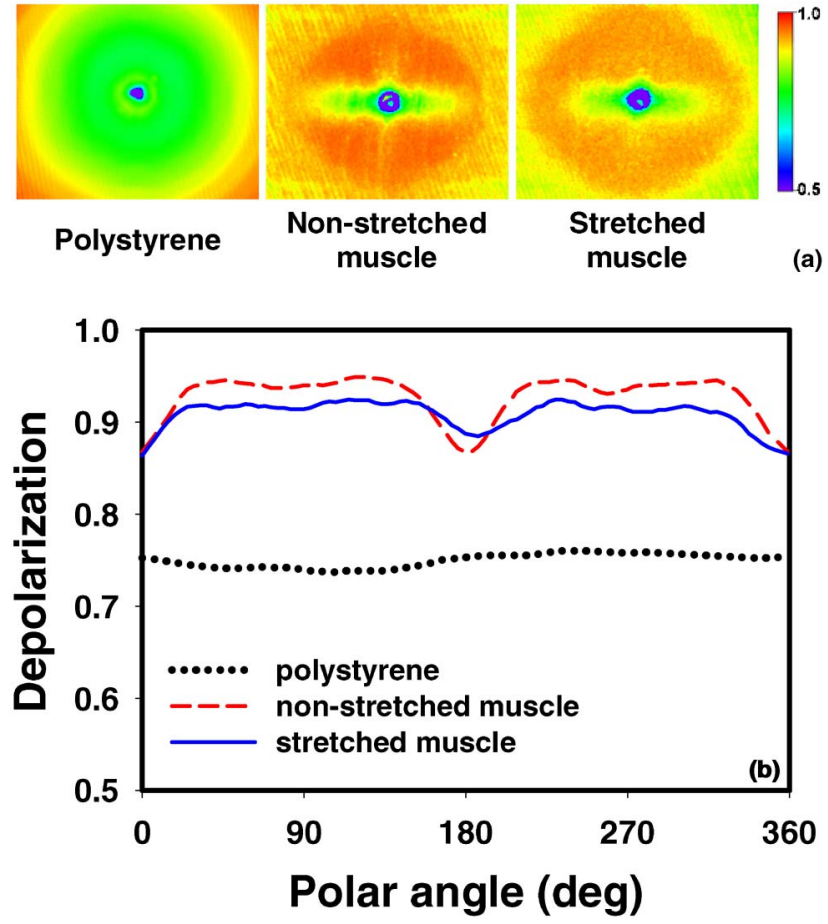

Fig. 5. (Color online) Depolarization images obtained in muscle and polystyrene solution. The depolarization values shown in (b) were extracted at locations $5 \mathrm{~mm}$ away from the incidence.

point, they have a high chance to be multiply scattered and thus have a high degree of depolarization.

The muscle sample shows a strong anisotropic depolarization pattern. A large depolarization $(>0.9)$ appears in the majority of the imaging area, which suggests that the muscle acts as an effective depolarizer. The depolarization capability can be attributed to the strong scattering properties from various muscle components, such as connective tissues and intramuscular fat. However, a small region along the $x$ axis (perpendicular to the muscle fibers) shows a smaller depolarization. This phenomenon is consistent with our previous observation [18] that the incident light maintains polarization better along the $x$ axis. Photons backscattered in this region likely experience fewer scattering events and are less depolarized.

The total retardance $R$ is a combination of linear retardance and optical rotation. The retardance results appear to be much noisier than the diattenuation and depolarization components. This is attributed to the increasing computation error in the late stage of the decomposition procedure because the retardance matrix is calculated after the other two components [21]. As shown in Fig. $\underline{6}$, no significant optical rotation exists in either skeletal muscle or polystyrene solution. Therefore, the measured retardance is primarily linear retardance. The decomposed total retardance in polystyrene solution is approximately $\pi$. This phase difference between the two orthogonal polarization directions is attributed to the reflection geometry used in our study. Because of this $\pi$ phase difference, a $45^{\circ}$ linearly polarized $(P)$ 


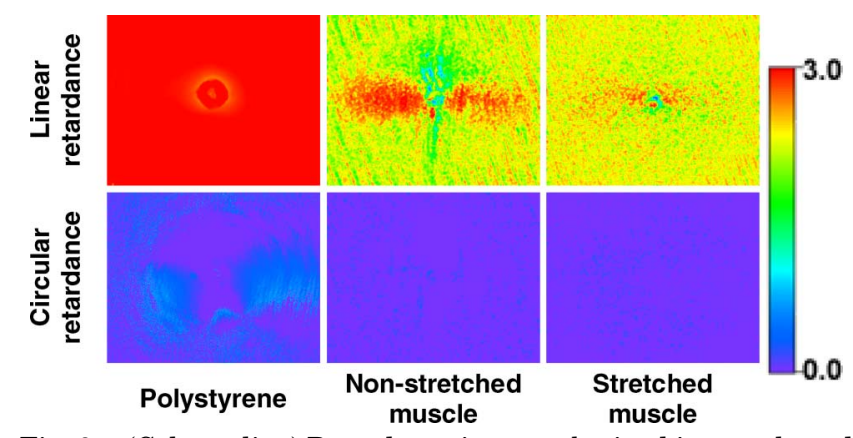

Fig. 6. (Color online) Retardance images obtained in muscle and polystyrene solution.

incident light becomes a $-45^{\circ}$ linearly polarized reflected light and right-hand circularly polarized $(R)$ incident light becomes left-hand circularly polarized reflected light.

In nonstretched muscle, the retardance is close to $\pi$ only within a small area along the $x$ axis. In other words, within the region perpendicular to muscle fibers, the muscle acts similarly as a backreflecting isotropic medium. Along the $y$ axis, the retardance values are smaller and approach $\pi / 2$ when close to the incident point. In all other areas, the retardance is between $\pi / 2$ and $\pi$. In stretched muscle, the retardance pattern along the $x$ and the $y$ axes becomes insignificant. Both scattering [11] and birefringence [22] can produce the retardance observed in muscle. However, further studies are necessary to clarify their contributions.

\section{Conclusion}

We applied the polar decomposition algorithm to analyze the reflectance polarization Mueller matrix in skeletal muscle samples. The extracted diattenuation $(D)$, retardance $(R)$, and depolarization $(\Delta)$ in muscle are very different from those obtained in an isotropic medium. The decomposed polarization images in muscle show strong anisotropic patterns along and/or perpendicular to muscle fibers, but rotationally symmetric patterns in the polystyrene solution. These anisotropic effects are related to the organized sarcomere structures in muscle. We found that stretching muscle along the fibers induced significant changes in the raw polarization-sensitive reflectance images. However, stretching induced minimal changes in the calculated Mueller matrix and the decomposed polarization images.

This work was supported in part by National Science Foundation (NSF) grant CBET-0643190.

\section{References}

1. R. R. Alfano, S. G. Demos, and S. K. Gayen, "Advances in optical imaging of biomedical media," Ann. N.Y. Acad. Sci. 820, 248-271 (1997).

2. J. R. Mourant, T. M. Johnson, S. Carpenter, A. Guerra, T. Aida, and J. P. Freyer, "Polarized angular dependent spectroscopy of epithelial cells and epithelial cell nuclei to determine the size scale of scattering structures," J. Biomed. Opt. 7, 378-387 (2002).
3. V. Backman, R. Gurjar, K. Badizadegan, I. Itzkan, P. R. Dasari, L. T. Perelman, and M. S. Feld, "Polarized light scattering spectroscopy for quantitative measurement of epithelial cellular structures in situ," IEEE J. Quantum Electron. 5, 1019-1026 (1999).

4. S. L. Jacques, J. R. Roman, and K. Lee, "Imaging superficial tissues with polarized light," Lasers Surg. Med. 26, 119 129 (2000).

5. W. S. Bickel, J. F. Davidson, D. R. Huffman, and R. Kilkson, "Application of polarization effects in light scattering: a new biophysical tool," Proc. Natl. Acad. Sci. USA 73, 486-490 (1976).

6. A. H. Hielscher, A. A. Eick, J. R. Mourant, D. Shen, J. P. Freyer, and I. J. Bigio, "Diffuse backscattering Mueller matrices of highly scattering media," Opt. Express 1, 441-453 (1997).

7. G. Yao and L.-H. Wang, "Two-dimensional depth-resolved Mueller matrix characterization of biological tissue by optical coherence tomography," Opt. Lett. 24, 537-539 (1999).

8. S. Lu and R. A. Chipman, "Interpretation of Mueller matrices based on polar decomposition,” J. Opt. Soc. Am. A 13, 11061113 (1996).

9. M. K. Swami, S. Manhas, P. Buddhiwant, N. Ghosh, A. Uppal, and P. K. Gupta, "Polar decomposition of $3 \times 3$ Mueller matrix: a tool for quantitative tissue polarimetry," Opt. Express 14, 9324-9337 (2006).

10. S. Manhas, M. K. Swami, P. Buddhiwant, N. Ghosh, P. K. Gupta, and K. Singh, "Mueller matrix approach for determination of optical rotation in chiral turbid media in backscattering geometry," Opt. Express 14, 190-202 (2006).

11. N. Ghosh, M. F. Wood, and I. A. Vitkin, "Mueller matrix decomposition for extraction of individual polarization parameters from complex turbid media exhibiting multiple scattering, optical activity, and linear birefringence," J. Biomed. Opt. 13, 044036 (2008).

12. M. H. Smith, "Interpreting Mueller matrix images of tissues," Proc. SPIE 4257, 82-89 (2001).

13. G. L. Liu, Y. Li, and B. D. Cameron, "Polarization-based optical imaging and processing techniques with application to the cancer diagnostics," Proc. SPIE 4617, 208-220 (2002).

14. J. Chung, W. Jung, M. J. Hammer-Wilson, P. Wilder-Smith, and Z. Chen, "Use of polar decomposition for the diagnosis of oral precancer," Appl. Opt. 46, 3038-3045 (2007).

15. P. Shukla, A. Awasthi, P. K. Pandey, and A. Pradhan, "Discrimination of normal and dysplasia in cervix tissue by Mueller matrix analysis," Proc. SPIE 6864, 686417 (2008).

16. J. Xia, A. Weaver, D. Gerrard, and G. Yao, "Monitoring sarcomere structure changes in whole muscle using diffuse light reflectance," J. Biomed. Opt. 11, 040504 (2006).

17. J. Ranasinghesagara and G. Yao, "Imaging 2D optical diffuse reflectance in skeletal muscle," Opt. Express 15, 3998-4007 (2007).

18. X. Li, J. Ranasinghesagara, and G. Yao, "Polarizationsensitive reflectance imaging in skeletal muscle," Opt. Express 16, 9927-9935 (2008).

19. R. Ossikovski, A. De Martino, and S. Guyot, "Forward and reverse product decompositions of depolarizing Mueller matrices," Opt. Lett. 32, 689-691 (2007).

20. J. Morio and F. Goudail, "Influence of the order of diattenuator, retarder, and polarizer in polar decomposition of Mueller matrices," Opt. Lett. 29, 2234-2236 (2004).

21. D. Hayes, "Error propagation in decomposition of Mueller matrices," Proc. SPIE 3121, 112-123 (1997).

22. J. J. Pasquesi, S. C. Schlachter, M. D. Boppart, E. Chaney, S. J. Kaufman, and S. A. Boppart, "In vivo detection of exercised-induced ultrastructural changes in genetically-altered murine skeletal muscle using polarization-sensitive optical coherence tomography," Opt. Express 14, 1547-1556 (2006). 